\title{
Inhibition of long non-coding RNA XIST upregulates microRNA-149-3p to repress ovarian cancer cell progression
}

Rong Jiang ${ }^{1}$, Hongyu Zhang ${ }^{1}$, Jinhua Zhou', Juan Wang ${ }^{1}$, Yuejuan $X u^{1}$, He Zhang ${ }^{1}$, Yanzheng Gu ${ }^{2,3,4}$, Fengqing Fu ${ }^{2,3,4}$, Yu Shen ${ }^{2,3,4}$, Guangbo Zhang ${ }^{2,3,4}$, Lanlan Feng ${ }^{5}$, Xueguang Zhang ${ }^{2,3,4}$, Youguo Chen ${ }^{1}$ and Fangrong Shen ${ }^{1}$

\begin{abstract}
Long non-coding RNAs (IncRNAs) and microRNAs (miRNAs) play critical roles in human diseases. We aimed to clarify the role of IncRNA X-inactive specific transcript (XIST)/miR-149-3p/forkhead box P3 (FOXP3) axis in ovarian cancer (OC) cell growth. XIST, miR-149-3p and FOXP3 expression in OC tissues and cell lines was assessed, and the predictive role of XIST in prognosis of OC patients was analyzed. The OC cell lines were screened and accordingly treated with silenced/overexpressed XIST plasmid or miR-149-3p mimic/inhibitor, and then the proliferation, invasion, migration, colony formation ability, apoptosis, and cell cycle distribution of OC cells were measured. Effect of altered XIST and miR-149-3p on tumor growth in vivo was observed. Online website prediction and dual luciferase reporter gene were implemented to detect the targeting relationship of IncRNA XIST, miR-149-3p, and FOXP3. XIST and FOXP3 were upregulated, whereas miR-149-3p was downregulated in OC tissues and cells. High XIST expression indicated a poor prognosis of OC. Inhibition of XIST or elevation of miR-149-3p repressed proliferation, invasion, migration, and colony formation ability, and promoted apoptosis and cell cycle arrest of HO-8910 cells. In SKOV3 cells upon treatment of overexpressed XIST or reduction of miR-149-3p, there exhibited an opposite tendency. Based on online website prediction, dual luciferase reporter gene, and RNA pull-down assays, we found that there was a negative relationship between XIST and miR-149-3p, and miR-149-3p downregulated FOXP3 expression. This study highlights that knockdown of XIST elevates miR-149-3p expression to suppress malignant behaviors of OC cells, thereby inhibiting OC development.
\end{abstract}

\section{Introduction}

Ovarian cancer $(\mathrm{OC})$ is the seventh commonest cancer all over the world in women and is the second most prevalent malignant tumor after breast cancer (BC) in women over the age of 40 years, especially in developed countries ${ }^{1}$. OC is a heterogeneous tumor that consists of

\footnotetext{
Correspondence: Youguo Chen (Youguochenschen@163.com) or Fangrong Shen (Shenfangrong22@163.com)

'Department of Obstetrics and Gynecology, The First Affiliated Hospital of Soochow University, Suzhou 215000 Jiangsu, China

2Jiangsu Institute of Clinical Immunology, The First Affiliated Hospital of Soochow University, Suzhou 215000 Jiangsu, China

Full list of author information is available at the end of the article

These authors contributed equally: Rong Jiang, Hongyu Zhang

Edited by $Y$. Shi
}

over 15 tumor types and subtypes based on histological features, and two-thirds of OC cases are diagnosed as high-grade serous types ${ }^{2}$. Despite increased survival rate during the recent decades, two-thirds of patients still die within 10 years of diagnosis. In women diagnosed with advanced-stage invasive epithelial OC, the 5-year survival rate is $<20 \%^{3}$. Primary cytoreductive surgery followed by platinum-based chemotherapy has been the cornerstone of treatment for OC patients. Despite improvements of the outcomes, current treatments become ineffective after several cycles of management and the recurrence rate is about $80-85 \%{ }^{4}$. Thus, it is urgently to explore novel targets for OC treatment.

\section{(c) The Author(s) 2021}

(c) (i) Open Access This article is licensed under a Creative Commons Attribution 4.0 International License, which permits use, sharing, adaptation, distribution and reproduction cc) in any medium or format, as long as you give appropriate credit to the original author(s) and the source, provide a link to the Creative Commons license, and indicate if changes were made. The images or other third party material in this article are included in the article's Creative Commons license, unless indicated otherwise in a credit line to the material. If material is not included in the article's Creative Commons license and your intended use is not permitted by statutory regulation or exceeds the permitted use, you will need to obtain permission directly from the copyright holder. To view a copy of this license, visit http://creativecommons.org/licenses/by/4.0/. 
Long non-coding RNAs (lncRNAs) are a cascade of RNA molecules with transcript lengths over 200 nucleotides and are able to regulate gene expression at epigenetic and transcriptional levels ${ }^{5}$. LncRNAs have been shown to be related with OC development. For instance, lncRNA HOTTIP has been reported to be a crucial indicator of $\mathrm{OC}$ prognosis and enhances cell proliferation and invasion ${ }^{6}$, and it has been unearthed that lncRNA FEZF1-AS1 promotes proliferation and inhibits apoptosis in $\mathrm{OC}^{7}$. LncRNA X-inactive specific transcript (XIST) is a $17 \mathrm{~kb}$ lncRNA that modulates X-chromosome inactivation of mammals to obtain gene dosage equivalence between XX female and XY male ${ }^{8}$. XIST has been considered to promote proliferation and differentiation of $\mathrm{OC}^{9}$ and it has also been demonstrated that XIST contributes to progression of cervical cancer ${ }^{10}$. MicroRNAs (miRNAs) are small non-coding RNA molecules able to posttranscriptionally regulate gene expression by targeting the $3^{\prime}$-untranslated region ( $3^{\prime}$-UTR) of mRNAs ${ }^{11}$. It has been unraveled that some particular miRNAs participate in OC progression, including miR-34 ${ }^{12}$ and miR$214^{13}$. As one of the miRNAs, miR-149 has been identified to suppress the proliferation and increase sensitivity of OC cells to cisplatin ${ }^{14}$, and miR-149-3p has been reported to be a novel biomarker for evaluating the prognosis of patients with epithelial $\mathrm{OC}$ and it might have potential therapeutic values ${ }^{15}$. Interestingly, the binding relationship between XIST and miR-149-5p has been confirmed in osteoarthritis ${ }^{16}$, whereas the correlation between miR149-3p and XIST in OC remains largely unknown. Forkhead box protein P3 (FOXP3) belongs to the forkhead-winged-helix family of transcriptional regulators. Moreover, it has been validated that FOXP3 is able to regulate cell proliferation, migration, and invasion in epithelial $\mathrm{OC}^{17}$, and FOXP has also been identified to be associated with poor prognosis in $\mathrm{OC}^{18}$. However, the relationship between miR-149-3p and FOXP3 remains to be explored. Therefore, we aimed to verify the role of lncRNA XIST/miR-149-3p/FOXP3 axis in OC progression and we inferred that altered XIST and miR-149-3p may regulate biological functions of OC cells, thereby affecting OC progression.

\section{Materials and methods \\ Ethics statement}

Written informed consents were acquired from all patients before this study. The protocol of this study was confirmed by the Ethic Committee of the First Affiliated Hospital of Soochow University. Animal experiments were strictly in accordance with the Guide to the Management and Use of Laboratory Animals issued by the National Institutes of Health. The protocol of animal experiments was approved by the Institutional Animal Care and Use Committee of the First Affiliated Hospital of Soochow University.

\section{Study subjects}

A total number of $45 \mathrm{OC}$ samples were collected from patients (aged 33-74 years, mean age of 52 years), who had accepted resection in the First Affiliated Hospital of Soochow University, and were all confirmed as OC by pathological diagnosis. According to the International Federation of Gynecology and Obstetrics (FIGO) stage ${ }^{19}$, there were 7 cases of I stage, 14 cases of II stage, and 24 cases of III stage. The collected tissues were placed in liquid nitrogen and preserved at $-80^{\circ} \mathrm{C}$ after $30 \mathrm{~min}$. The patients had not accepted any treatment before the resection. Forty normal ovarian tissues and 22 pairs of non-metastatic lymph nodes and lymph node metastatic tumors were also collected. Complete follow-up data of the patients were obtained through outpatient service and telephone. The follow-up period ended on 30 April 2019 and lasted for 60 months.

\section{Cell culture and screening}

Human normal ovarian epithelial cells (NOECs) and human epithelial OC cells (OVCAR3, CAOV3, SKOV3, HO-8910, and HO-8910PM) were all purchased from Shanghai Institute of Biochemistry and Cell Biology, Chinese Academy of Sciences (Shanghai, China). NOECs were trypsinized for primary culture and placed in keratinocyte serum-free medium containing $100 \mathrm{U} /$ $\mathrm{mL}$ penicillin and $100 \mu \mathrm{g} / \mathrm{mL}$ streptomycin for continuous subculture; SKOV3, HO-8910, and HO8910PM cells were cultured with Roswell Park Memorial Institute (RPMI)-1640 medium containing $100 \mathrm{~mL} / \mathrm{L}$ fetal bovine serum (FBS); OVCAR3 cells were cultured by RPMI- 1640 medium containing $200 \mathrm{~mL} / \mathrm{L}$ FBS and $155 \mathrm{U} / \mathrm{L}$ insulin; CAOV3 cells were cultured with Dulbecco's modified Eagle medium containing $100 \mathrm{~mL} / \mathrm{L}$ FBS. All of the above culture solutions contained $100 \mathrm{U} / \mathrm{mL}$ penicillin and $100 \mu \mathrm{g} / \mathrm{mL}$ streptomycin. HO-8910 cells had the largest, whereas SKOV3 cells had the least difference in corresponding expression from NOECs; hence, the two OC cell lines were screened for subsequent experiments.

\section{Cell grouping and transfection}

SKOV3 and HO-8910 cells were seeded onto a 24-well plate at $1 \times 10^{5}$ cells/well and then transfected after $80 \%$ cell confluence. The transfection was in line with protocols of lipofectamine 2000 reagent (Invitrogen, Inc., CA, USA) and the relative sequences were obtained from GenePharma Co., Ltd (Shanghai, China).

SKOV3 cells were divided into seven groups and respectively transfected with overexpressed (OE)-XIST, miR-149-3p inhibitor, OE-XIST + miR-149-3p mimic, or their negative controls (NCs).

HO-8910 were also classified into seven groups and were respectively transfected with short hairpin RNA 
(sh)-XIST, miR-149-3p mimic, sh-XIST + miR-149-3p inhibitor, or their NCs.

\section{Reverse-transcription quantitative PCR}

Total RNA in tissues and cells was extracted with RNeasy Plus Mini Kit (QIAGEN company, Hilden, Germany). Reverse transcription was conducted by TaqMan MicroRNA Reverse Transcription Kit (Applied Biosystems, Inc., CA, USA) and the reaction solution was used for fluorescent quantitative PCR (qPCR) based on directions of SYBR Premix Ex Taq ${ }^{\mathrm{TM}}$ II kits (Takara Bio, Inc., Otsu, Shiga, Japan) on a BIO-RAD CFX96 instrument. The primers (Table 1) were designed by Shanghai Sangon Biotechnology Co., Ltd (Shanghai, China). U6 was used as the loading control of miR-149-3p and glyceraldehyde phosphate dehydrogenase (GAPDH) was used as the loading control of XIST and FOXP3. The data were analyzed by $2^{-\triangle \triangle \mathrm{Ct}}$ method.

\section{Western blot analysis}

Total protein was extracted from OC tissues and cells and the protein concentration was measured. The proteins were conducted with $10 \%$ SDS-polyacrylamide gel electrophoresis and transferred onto membranes, which were then blocked with $5 \%$ skim milk powder at $4{ }^{\circ} \mathrm{C}$ overnight. Subsequently, the membranes were probed with primary antibody against FOXP3 $(1: 1000$, Invitrogen, Inc.) and GAPDH $(1: 1000$, Santa Cruz Biotechnology, Inc., CA, USA) overnight, and then were re-probed with horseradish peroxidase-conjugated immunoglobulin G $(1: 1000$, BOSTER Biological Technology Co., Ltd, Wuhan, Hubei, China) secondary antibody at $37^{\circ} \mathrm{C}$ for $1 \mathrm{~h}$, followed by soaked in enhanced chemiluminescent reaction solution (Pierce, Rockford, IL, USA) for $1 \mathrm{~min}$. With the fluid removed, the membranes were covered by

Table 1 Primer sequence.

\begin{tabular}{ll}
\hline Gene & Primer sequence $\left(\mathbf{5}^{\prime} \mathbf{- 3}^{\prime} \mathbf{)}\right.$ \\
\hline XIST & Forward: CGGGTCTCTTCAAGGACATTTAGCC \\
& Reverse: GCACCAATACAGAGGAATGGAGGG \\
MiR-149-3p & Forward: ACACTCCAGCTGGGAGGGGGGACGGGGGC \\
& Reverse: CTCAACTGGTGTCGTGGA \\
FOXP3 & Forward: ATCGCTGCTAGCTACTTAGCTA \\
& Reverse: CTGATCGTGAACTGCCGTGGCTA \\
GAPDH & Forward: GAAGATGGTGATGGGATTTC \\
& Foverse: GAAGGTGAAGGTCGGAGT \\
U6 & Reverse: AACGCTTCACGAATTTGCGT \\
\hline
\end{tabular}

FOXP3 forkhead box P3, GAPDH glyceraldehyde phosphate dehydrogenase, miR149-3p microRNA-149-3p, XIST X-inactive specific transcript. food wrap and exposed in the dark, then developed, fixed, and observed. GAPDH was taken as the internal reference and the protein bands were analyzed using Image $2 x$ software.

\section{MTT assay}

Cells in each group were trypsinized, seeded, and cultured for $0,12,24,36$, and $48 \mathrm{~h}$, respectively. The cell viability was performed with MTT (3-(4,5-dimethyl-2thiazolyl)-2,5-diphenyl-2-H-tetrazolium bromide) assay as previously described $^{20}$. The optical density at $490 \mathrm{~nm}$ of each well was analyzed by a microplate reader (Anthos Labtec, Wals, Austria).

\section{Colony formation assay}

Cells in each group that had been cultured for $24 \mathrm{~h}$ were trypsinized and seeded onto $35 \mathrm{~mm}$ culture dishes for 10day culture and the medium was changed every 3 days. After this, the cells were fixed with $40 \mathrm{~g} \mathrm{~L}^{-1}$ paraformaldehyde and stained by $1 \mathrm{~g} \mathrm{~L}^{-1}$ crystal violet dye solution for $20 \mathrm{~min}$. The number of colonies ( $>50$ cells) was counted under a microscope.

\section{Flow cytometry \\ Determination of cell cycle distribution}

SKOV3 and HO-8910 cells that had been transfected for $48 \mathrm{~h}$ were trypsinized and resuspended by phosphatebuffered saline (PBS). The cell suspension was centrifuged at 2000 r.p.m. for $5 \mathrm{~min}$ with the supernatant discarded and there was a backflow of $50 \mu \mathrm{L}$ remaining PBS. The cells were resuspended by flicking the tubes and appended with $2 \mathrm{~mL}$ PBS, and then centrifuged at 2000 r.p.m. for $5 \mathrm{~min}$ with the supernatant removed. The cell concentration was adjusted to $1 \times 10^{6}$ cells/mL by adding $1 \times$ binding buffer and the cells were added with $1 \mathrm{~mL}$ PBS, $20 \mu \mathrm{L}$ propidium iodide (PI), $5 \mu \mathrm{L}$ RNase, and $1 \mu \mathrm{L} 20 \%$ Nonidet P40, then transferred into the flow tubes. Each sample was added with $300-500 \mu \mathrm{L}$ labeling liquid for 3-5 min and the cell cycle distribution was determined.

\section{Detection of apoptosis}

SKOV3 and HO-8910 cells that had been transfected for $48 \mathrm{~h}$ were centrifuged and transferred into $2.0 \mathrm{~mL}$ Eppendorf tubes, which were fixed with $1 \mathrm{~mL}$ absolute ethanol at $-20^{\circ} \mathrm{C}$ for over $24 \mathrm{~h}$. After centrifugation, the cells were resuspended with $1 \mathrm{~mL} 5 \%$ PBS and rinsed again. The sediment was suspended with $80 \mu \mathrm{L} 1 \mathrm{mg} / \mathrm{mL}$ Rnase A and placed at $37^{\circ} \mathrm{C}$ for $30 \mathrm{~min}$, then appended with $400 \mu \mathrm{L} 50 \mu \mathrm{g} / \mathrm{mL}$ PI in dark for $10 \mathrm{~min}$. The apoptosis of SKOV3 and HO-8910 cells was measured.

\section{Dual luciferase reporter gene assay}

A biological online prediction website (https://cm. jefferson.edu/rna22/Precomputed/) was used to predict 
the potential binding sites of miR-149-3p and XIST. XIST sequence was obtained and the sequence segments was synthetized by PCR amplification with genomic DNA as the template. The segment was digested and purified, then connected with pGL3-Control vector by T4 ligase and transformed. After the identification of positive colony, the sample was seeded into Luria-Bertani medium containing ampicillin and incubated at $37^{\circ} \mathrm{C}$ for $12-16 \mathrm{~h}$. Afterwards, agarose gel electrophoresis, sequencing, and comparative analysis were successively performed. MiR149-3p-binding site mutant (MUT) vector of pGL3-XIST was synthetized by Wuhan GeneCreate Biological Engineering Co., Ltd (Hubei, China). Wild-type (WT) plasmid containing target sequence was named as XIST-WT and MUT type plasmid that had undergone site-directed mutagenesis was defined as XIST-MUT. SKOV3 and HO8910 cells were detached, seeded, and incubated until cell confluence reached $60 \%$. The transfection was in line with instructions of X-tremegene HP transfection reagent (Roche, Ltd, Basel, Switzerland): transfection reagent and XIST-WT or XIST-MUT with miR-150-3p mimic, or its $\mathrm{NC}$ were added into opti-minimum essential medium for $20 \mathrm{~min}$, and then appended onto cell culture plates and incubated for $6 \mathrm{~h}$. Each well was supplemented with $200 \mu \mathrm{L}$ complete medium containing 10\% FBS. The medium was removed after $48 \mathrm{~h}$ incubation and the cells were reacted with $300 \mu \mathrm{L} 1 \times$ passive lysis buffer at $4{ }^{\circ} \mathrm{C}$ for $20 \mathrm{~min}$. Subsequently, $40 \mu \mathrm{L}$ cell lysis buffer was appended on the Lockwell maxisorp plates and added with $20 \mu \mathrm{L}$ luciferase assay reagent, followed by detection of firefly luciferase activity by a microplate reader. Fifty microliters of Stop\&Glo ${ }^{\circledR}$ reagent was supplemented into each well and the Renilla luciferase activity was gauged by a microplate reader. Finally, the relative luciferase activity of each sample was calculated and data were analyzed.

A bioinformatic online website (https://cm.jefferson.edu/ rna22/Precomputed/) was utilized to predict the target relation between miR-149-3p and FOXP3, and binding sites of miR-149-3p and FOXP3 3'-UTR. FOXP3 3'-UTR promoter sequence containing binding sites of miR-149-3p was synthetized. FOXP3 $3^{\prime}$-UTR WT plasmid (FOXP3-WT) and FOXP3 3'-UTR MUT plasmid (FOXP3-MUT) were constructed by Wuhan GeneCreate Biological Engineering. SKOV3 and HO-8910 cells were detached, seeded, cultured, and transfected. The luciferase activity of each sample was determined and the relative luciferase activity was calculated as luciferase activity rate of Renilla/firefly.

\section{Subcutaneous tumorigenesis in nude mice}

Forty-two male nude mice aged 4-6 weeks old (Experimental Animal Center, Yunnan University, Yunan, China) were randomly classified into 14 groups and were subcutaneously injected with $0.1 \mathrm{~mL}$ OC cell suspension according to the cell grouping. Three mice in each group were placed in the same breeding box and kept in an specific pathogen free (SPF) animal laboratory. Nude mice need to adapt to the environment in an SPF animal laboratory for a week, during which time they should change their feed, bedding, and water bottles, and their health status were observed daily. One week later, each group of cultured OC cells were made into cell suspension and placed in cell cryotubes, and $0.1 \mathrm{~mL}$ of cell suspension $\left(1 \times 10^{6}\right)$ per mouse was injected under the skin of the back of the neck. Before injection, $75 \%$ alcohol should be used to disinfect the injection site. After injection, the injection site was pressed with a sterile cotton swab to prevent cell suspension from flowing out of the needle well. The tumor growth was observed after 3-5 days of the injection. Nude mice were normally weighed and tumor volume was measured. On the day 25 of injection, the nude mice were killed and the xenografts were collected and weighed.

\section{Statistical analysis}

All data analyses were conducted using SPSS 21.0 software (IBM, Corp., Armonk, NY, USA). The measurement data were expressed as mean $\pm S D$. The $t$-test was performed for comparisons between two groups, one-way analysis of variance (ANOVA) was used for comparisons among multiple groups and Tukey's post hoc test was used for pairwise comparisons after one-way ANOVA. Kaplan-Meier was used to analyze the prognosis of OC patients. $P$-value $<0.05$ was indicative of statistically significant difference.

\section{Results}

XIST and FOXP3 are upregulated, whereas miR-149-3p is downregulated in OC tissues, and high expression of XIST indicates a poor prognosis of OC patients

Levels of XIST, miR-149-3p, and FOXP3 were determined by reverse-transcription qPCR (RT-qPCR) and western blot assay, and we found that (Fig. 1A-E) XIST and FOXP3 expression was upregulated, whereas miR149-3p expression was downregulated in OC tissues vs. those in normal ovarian tissues.

OC patients were grouped based on FIGO stage and XIST expression in patients of different stages was evaluated by RT-qPCR. It came out that (Fig. 1F, G) XIST expression in patients at advanced stages was higher than those at early stages, whereas there showed no noticeable difference in XIST expression among patients of difference ages. The detection results of XIST expression in 22 pairs of nonmetastatic lymph nodes and metastatic lymph nodes showed that in comparison to non-metastatic lymph nodes, XIST expression was elevated in metastatic lymph nodes (Fig. $1 \mathrm{H}$ ). Based on the median of relative expression of XIST, OC patients were divided into high and low expression groups, and the predictive role of XIST in prognosis of OC patients was evaluated by Kaplan-Meier analysis, and the findings (Fig. 1I) suggested that high XIST expression was associated with a poor prognosis of OC. 

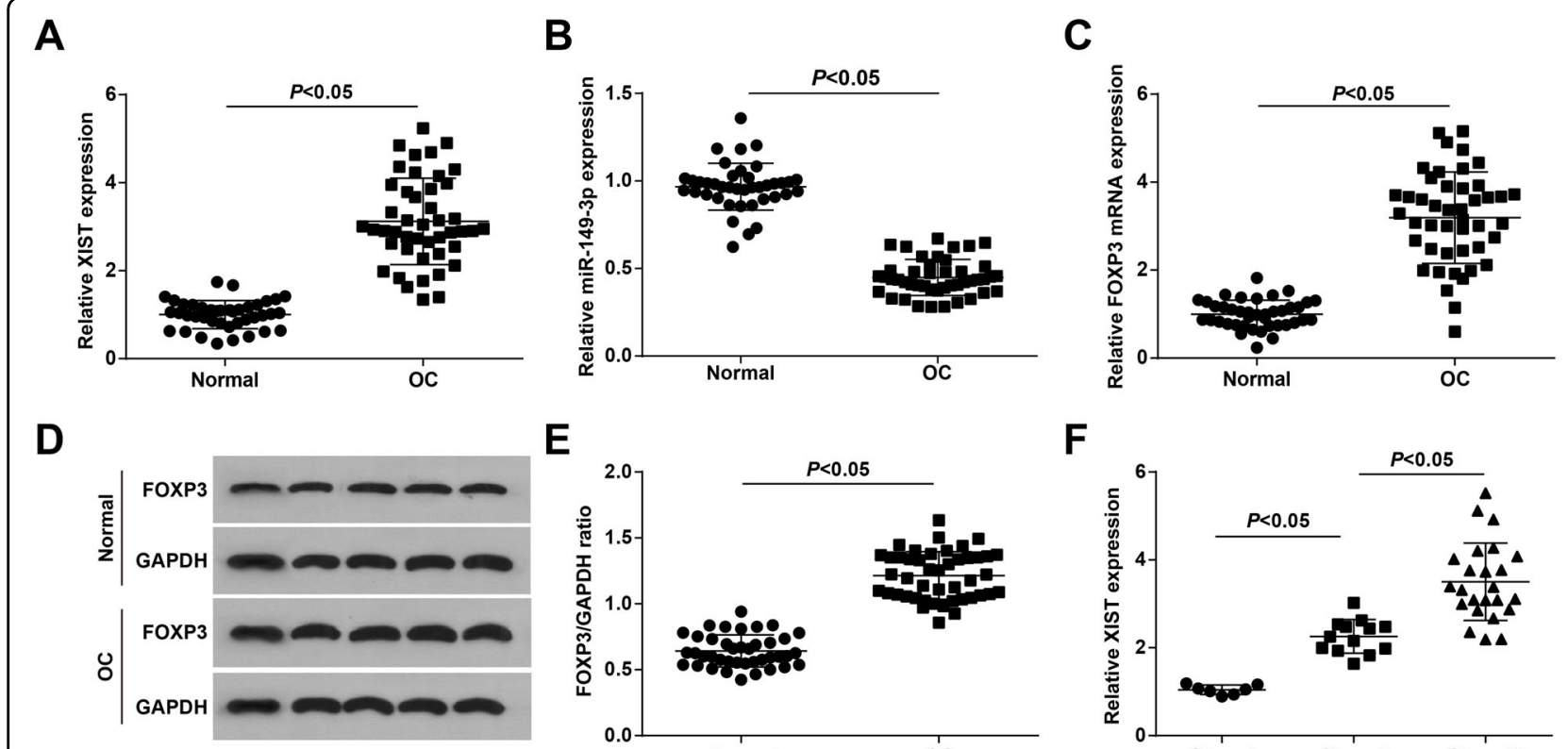

\section{E \\ H}

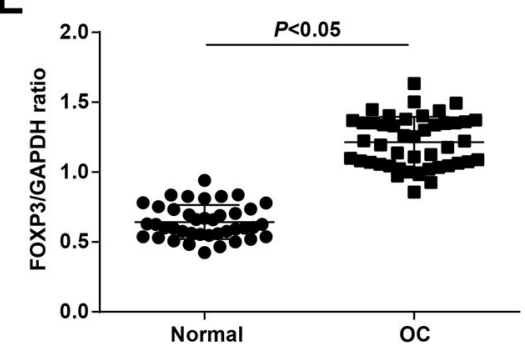

$\mathbf{F}$
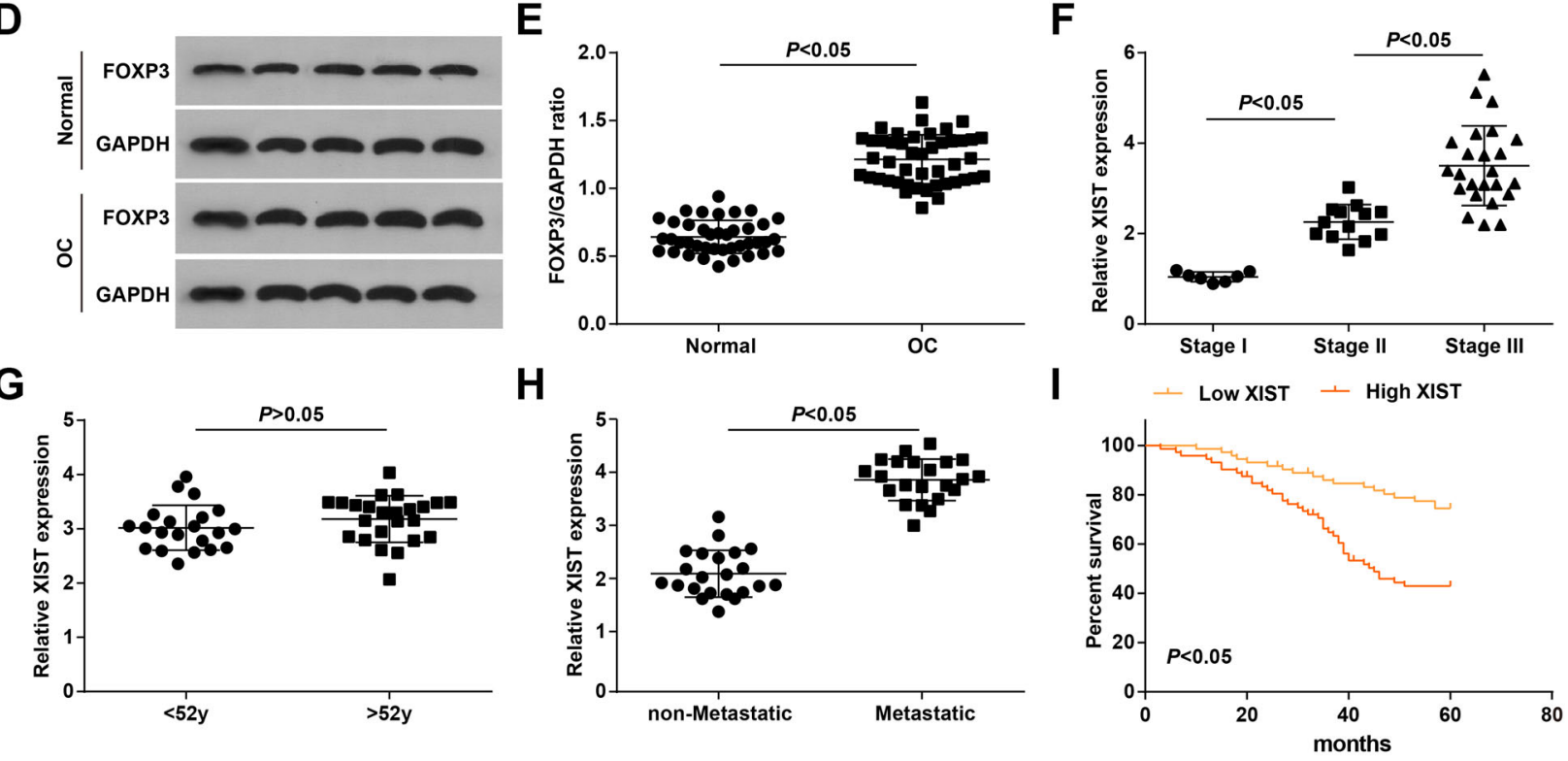

Fig. 1 XIST and FOXP3 are upregulated, whereas miR-149-3p is downregulated in OC tissues, and high expression of XIST indicates a poor prognosis of OC patients. A XIST expression in normal ovarian tissues and OC tissues. B miR-149-3p expression in normal ovarian tissues and OC tissues. C FOXP3 mRNA expression in normal ovarian tissues and OC tissues. D Protein band of FOXP3 in normal ovarian tissues and OC tissues. E Protein expression of FOXP3 in normal ovarian tissues and OC tissues. F Impact of FIGO stage on XIST expression. G Impact of age on XIST expression. $\mathbf{H}$ Impact of LNM on XIST expression. I Predictive role of XIST in prognosis of OC patients. The measurement data were expressed as mean \pm SD, $t$-test was performed for comparisons between two groups, and Kaplan-Meier analysis was used to analyze the prognosis of OC patients.

\section{Expression and transfection efficiency identification of} XIST and FOXP3 in OC cell lines

To explore the effect of XIST and miR-146-3p on XIST, miR-149-3p, and FOXP3 expression in SKOV3 and HO8910 cells, XIST, miR-149-3p, and FOXP3 expression in OC cell lines and NOECs were determined by RT-qPCR and western blot assay, and it was observed that (Fig. 2A-E) OC cell lines had higher XIST and FOXP3 expression, and lower miR-149-3p expression vs. NOECs. Among the OC cell lines, SKOV3 cells had the least, whereas HO-8910 cells had the largest difference in XIST and FOXP3 expression from NOECs.

SKOV3 cells were transfected with OE-XIST, miR-149-3p inhibitor, OE-XIST + miR-149-3p mimic, or their NCs, and HO-8910 were transfected with sh-XIST, miR-149-3p mimic, sh-XIST + miR-149-3p inhibitor, or their NCs, to investigate the effects of XIST and miR-149-3p on OC cells.
RT-qPCR was used to assess the transfection efficiency. The results suggested that in SKOV3 cells (Fig. 2F, G), transfection of OE-XIST and the transfection of miR-149-3p inhibitor inhibited miR-149-3p expression; in HO-8910 cells (Fig. $2 \mathrm{H}, \mathrm{I})$, treatment of sh-XIST reduced XIST expression and treatment of miR-149-3p mimic upregulated miR-149-3p.

Inhibited XIST or elevated miR-149-3p represses proliferation and promotes apoptosis of OC cells, and arrests OC cells at G0/G1 phase

Proliferation and colony formation ability of $\mathrm{OC}$ cells were determined by MTT and colony formation assay. The results indicated that in SKOV3 cells (Fig. 3A, C, D), proliferation and colony formation ability of cells were promoted by treatment of OE-XIST or miR-149-3p inhibitor; the effect of OE-XIST on proliferation and colony formation ability of SKOV3 cells was reversed by miR-149-3p mimic. 


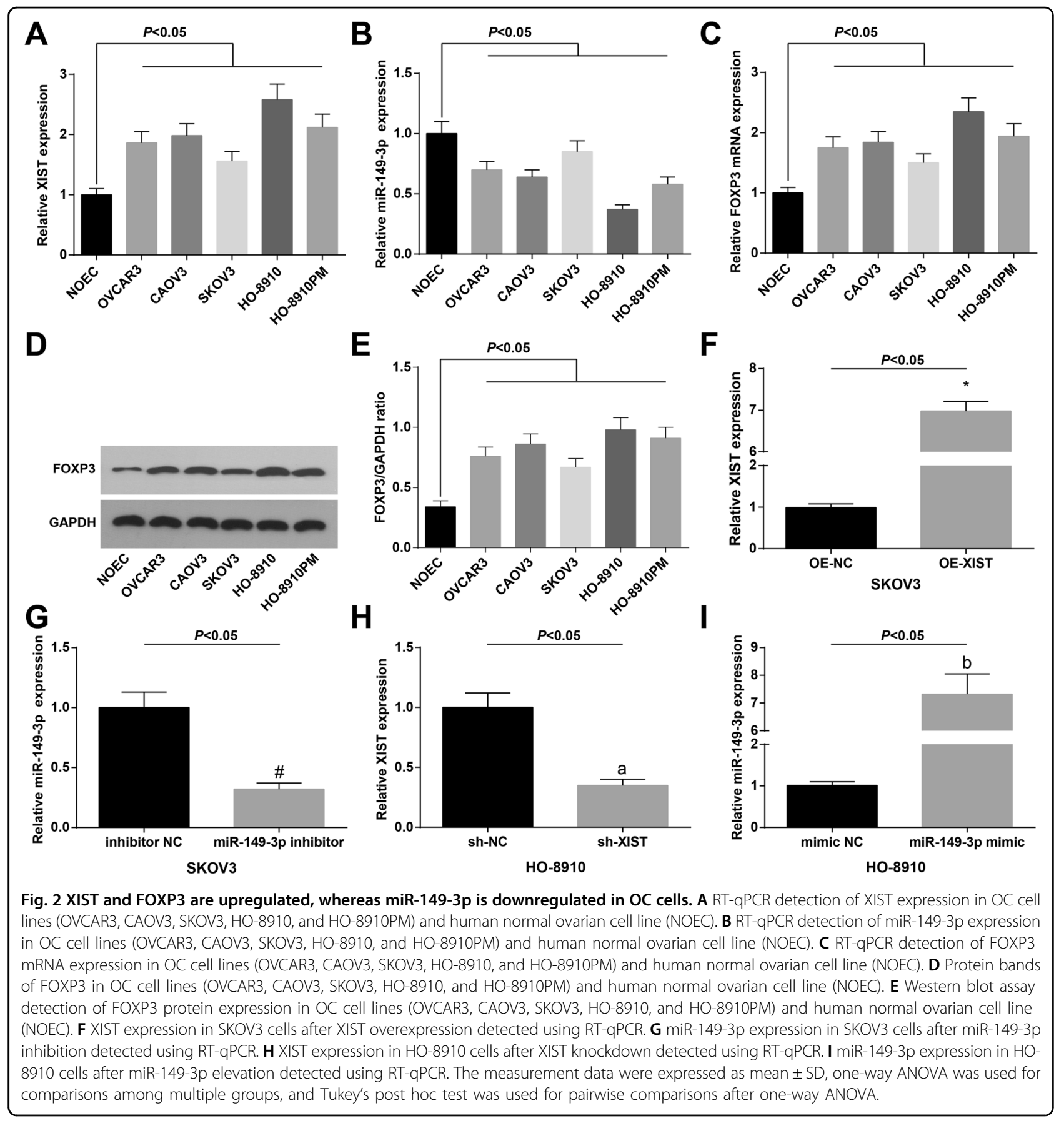

In HO-8910 cells (Fig. 3B, E, F), proliferation and colony formation ability of cells were repressed by treatment of sh-XIST or miR-149-3p mimic; miR-149$3 p$ inhibitor abolished the impact of sh-XIST on proliferation and colony formation ability of $\mathrm{HO}$ 8910 cells.

Apoptosis and cell cycle distribution were measured by flow cytometry and we found that in SKOV3 cells (Fig. 4A-D), OE-XIST or miR-149-3p inhibitor arrested cells in $\mathrm{S}$ and G2/M phases, and reduced the apoptosis rate of SKOV3 cells; the effects of OE-XIST on cell cycle arrest and apoptosis rate of SKOV3 cells were reversed by miR149-3p mimic.

In HO-8910 cells (Fig. 4E-H), sh-XIST or miR-149-3p mimic arrested cells in G0/G1 phase and augmented apoptosis rate $\mathrm{HO}-8910$ cells; the effects of sh-XIST on cell cycle arrest and apoptosis rate of HO-8910 cells were reversed by miR-149-3p inhibitor. 

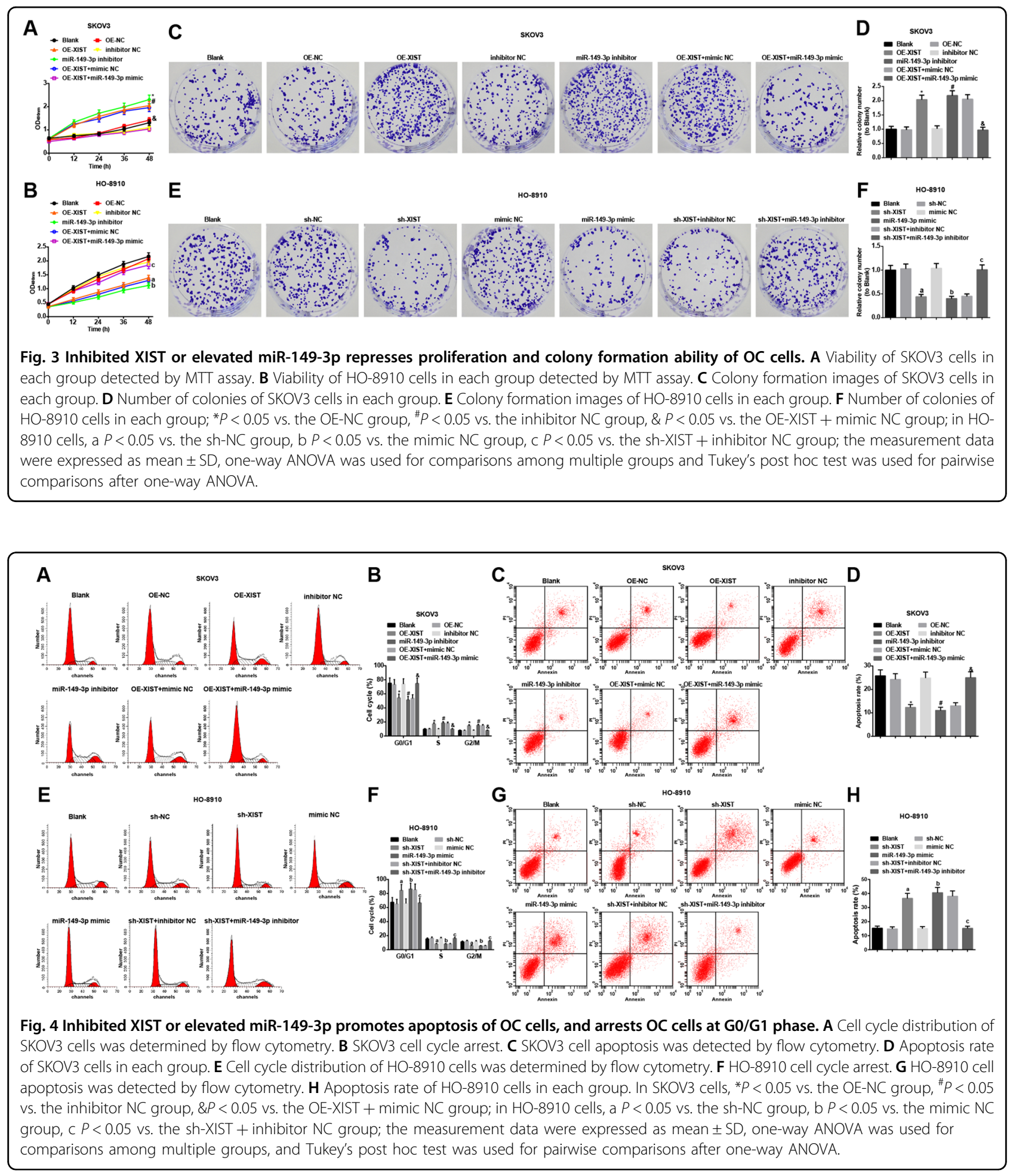

\section{Inhibited XIST or elevated miR-149-3p suppresses migration and invasion of $O C$ cells}

Invasion and migration abilities of OC cells were measured by Transwell assay and we found that in SKOV3 cells (Fig. 5A, B), the invasive and migratory cells were increased by OE-XIST or miR-149-3p inhibitor; miR-149$3 \mathrm{p}$ mimic abrogated the impact of OE-XIST on invasion and migration abilities of SKOV3 cells.

In $\mathrm{HO}-8910$ cells (Fig. 5C, D), the invasive and migratory cells were decreased by sh-XIST or miR-149-3p 
mimic; effects of sh-XIST on invasion and migration abilities of HO-8910 cells were abolished by miR-149-3p inhibitor.

\section{There is a negative relationship between miR-149-3p and XIST}

LncRNAs are known to function through their interaction with downstream target miRNAs ${ }^{21}$. To identify whether XIST could interact with miRNA to regulate the OC cell proliferation and tumor growth, we searched for the bioinformatic website (https://cm.jefferson.edu/ rna22/) to predict that there existed binding sites between XIST and miR-149-3p (Fig. 6A). The expression of miR-149-3p in SKOV3 and HO-8910 cells was assessed using RT-qPCR after XIST was knocked down or upregulated, and we found that in SKOV3 cells, XIST overexpression inhibited miR-149-3p expression; in HO-8910 cells, XIST reduction increased miR-149-3p expression (Fig. 6B). Then, we regulated miR-149-3p expression to verify whether miR-149-3p could affect XIST expression. As shown in Fig. 6C, in SKOV3 cells, XIST was OE after miR-149-3p was downregulated; in HO-8910 cells, XIST was reduced after miR-149-3p was upregulated. The dual luciferase reporter gene assay was performed to further confirm the direct binding relationship between XIST and miR-149-3p. The results revealed that in SKOV3 and HO8910 cells, the co-transfection of FOXP3-WT and miR- 149-3p mimic decreased the luciferase activity, which was not affected by the co-transfection of FOXP3-MUT and miR-149-3p mimic (Fig. 6D, E). To explore whether there existed a negative relationship between XIST and miR149-3p, RT-qPCR was conducted in 45 OC tissues. The results indicated that the expression levels of XIST and miR-149-3p were in a negative correlation $(r=-0.644, P<$ 0.001, Fig. 6F). These data suggested that XIST and miR149-3p negatively regulated each other in OC.

FOXP3 is a forkhead transcription factor and miRNAs can directly bind to FOXP3 $3^{\prime}$-UTR and inhibit its expression $^{22}$. First, a bioinformatic website confirmed the potential binding sites of miR-149-3p in FOXP3 3 '-UTR (Fig. 6G). The results of dual luciferase reporter gene assay showed that (Fig. 6H, I) after SKOV3 and HO-8910 cells were co-transfected with FOXP3-WT and miR-149$3 \mathrm{p}$ mimic, the relative luciferase activity of SKOV3 and HO-8910 cells was decreased significantly, whereas the co-transfection of FOXP3-MUT and miR-149-3p did not affect the relative luciferase activity of SKOV3 and HO8910 cells.

In addition, RT-qPCR and western blot analysis showed that overexpression of XIST or inhibition of miR-149-3p significantly increased FOXP3 mRNA and protein expression in SKOV3 cells, and the upregulation of miR149-3p could reduce OE-XIST-induced FOXP3 expression (Fig. 6J, K); in HO-8910 cells, the knockout of XIST

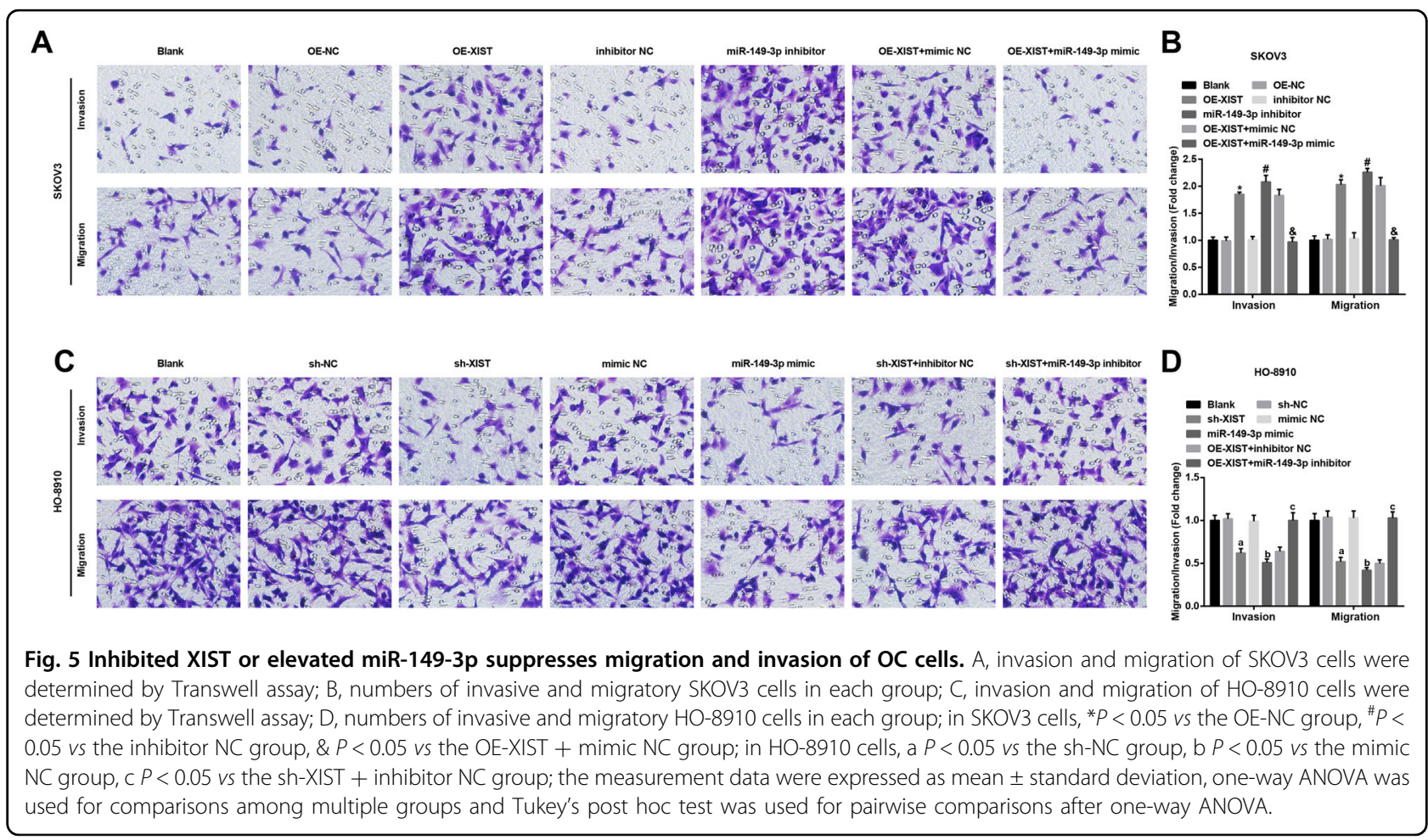




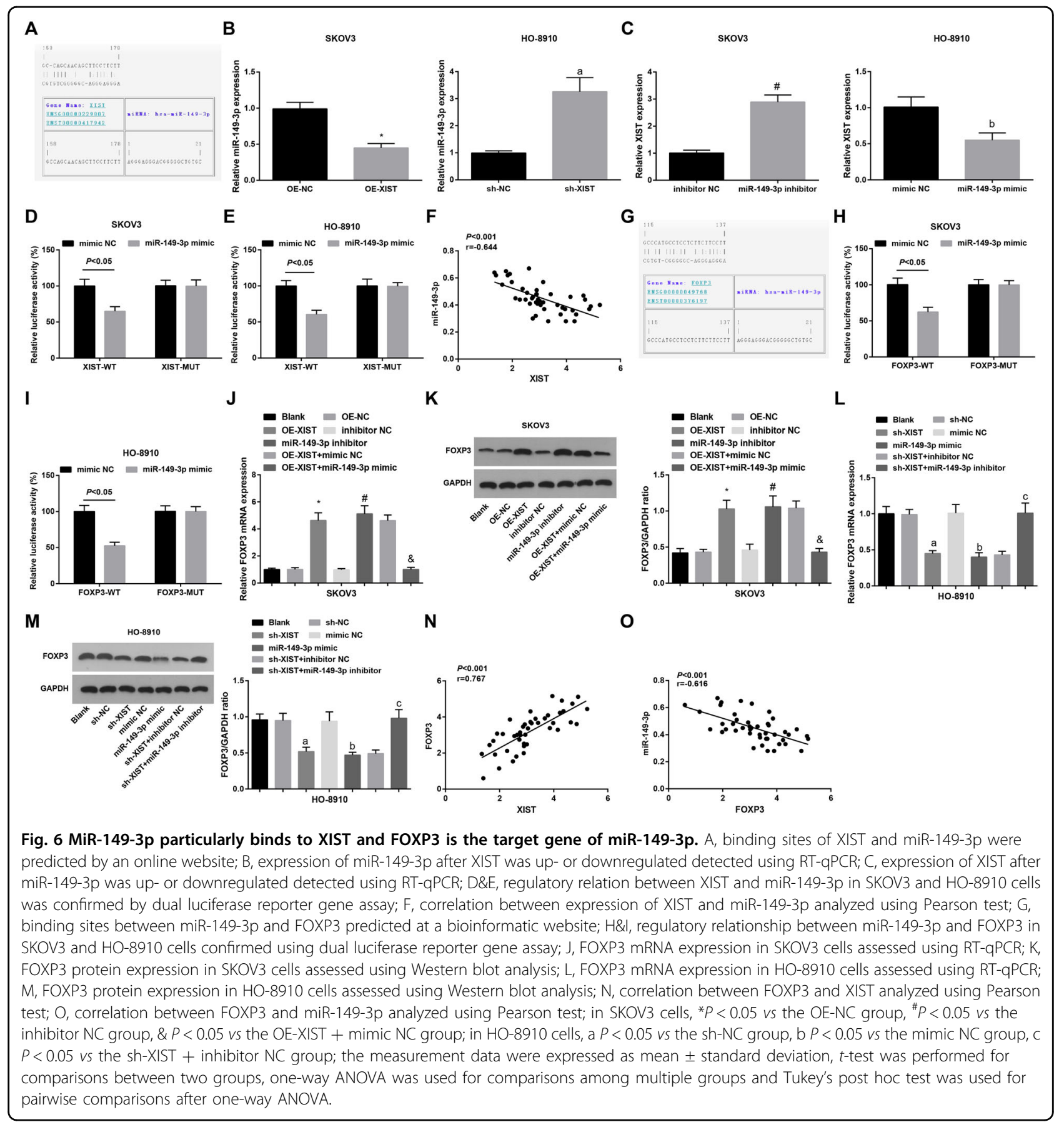

or elevation of miR-149-3p significantly decreased FOXP3 mRNA and protein expression, whereas miR-149-3p inhibitor elevated the sh-XIST-induced FOXP3 mRNA and protein expression (Fig. 6L, M). Finally, Pearson's test showed that FOXP3 was positively correlated with XIST $(r=0.767, P<0.001$, Fig. $6 \mathrm{~N})$ and FOXP3 was negatively correlated with miR-149-3p $(r=-0.616, P<0.001$, Fig. $6 \mathrm{O})$. These results indicated that FOXP3 is a direct target gene of miR-149-3p and XIST can regulate FOXP3 in OC cells by regulating miR-149-3p.

\section{Inhibited XIST or elevated miR-149-3p restricts ovarian tumor growth in vivo}

Results of subcutaneous tumorigenesis in nude mice mirrored that in SKOV3 xenografts (Fig. 7A-C), tumor weight and volume were both increased by OE-XIST or 
miR-149-3p inhibitor; effect of OE-XIST on tumor weight and volume was reversed by miR-149-3p mimic.

In HO-8910 xenografts (Fig. 7D-F), tumor weight and volume were reduced by sh-XIST or miR-149-3p mimic; impacts of sh-XIST on tumor weight and volume were abrogated by miR-149-3p inhibitor.

\section{Discussion}

Among all types of malignancies, $\mathrm{OC}$ is a leading cause of cancer-correlated death in women and the most fatal gynecologic cancer ${ }^{1}$. According to the competing endogenous RNA hypothesis (also known as the sponge function), IncRNAs are capable of regulating gene expression through titrating miRNAs to participate in various biological processes $^{23}$. We focused on the role of lncRNA XIST/miR-1493p/FOXP3 axis in OC progression and we found that inhibition of IncRNA XIST could bound with miR-149-3p to repress malignant episodes of OC cells.

We determined the expression levels of XIST, miR-149-3p, and FOXP3 in OC tissues and cells. The results implied that XIST and FOXP3 were upregulated, whereas miR-149-3p was downregulated in both $\mathrm{OC}$ tissues and cell lines, respectively, compared with normal ovarian tissues and NOECs. Consistently, Zhu et al. ${ }^{24}$ have discovered that XIST is $\mathrm{OE}$ in cervical cancer tissues and cell lines, and this overexpression is also demonstrated by another study focusing on cervical cancer ${ }^{10}$. Moreover, miR-149-3p expression has been demonstrated to be repressed in tumor-bearing mouse spleens in $\mathrm{BC}^{25}$ and Sun et al. ${ }^{14}$ have supported that miR-149 is decreased in OC tissues and cells. A higher expression of FOXP3 in epithelial OC tissues at advanced stages has been found in a recent study ${ }^{26}$. Besides, we verified that the high expression of XIST indicated a poor prognosis of OC patients and the predictive role of XIST in prognosis of $\mathrm{BC}$ patients has been identified as well ${ }^{27}$. In addition, we found through online prediction, dual luciferase reporter gene assay, and RNA pull-down assay that XIST could bind to miR-149-3p, and FOXP3 was confirmed as a direct target of miR-149-3p. Consistently, the binding relationship between XIST and miR-149-5p has been identified in osteoarthritis ${ }^{16}$, whereas the targeting relationship between miR-149-3p and FOXP3 remains largely unknown.

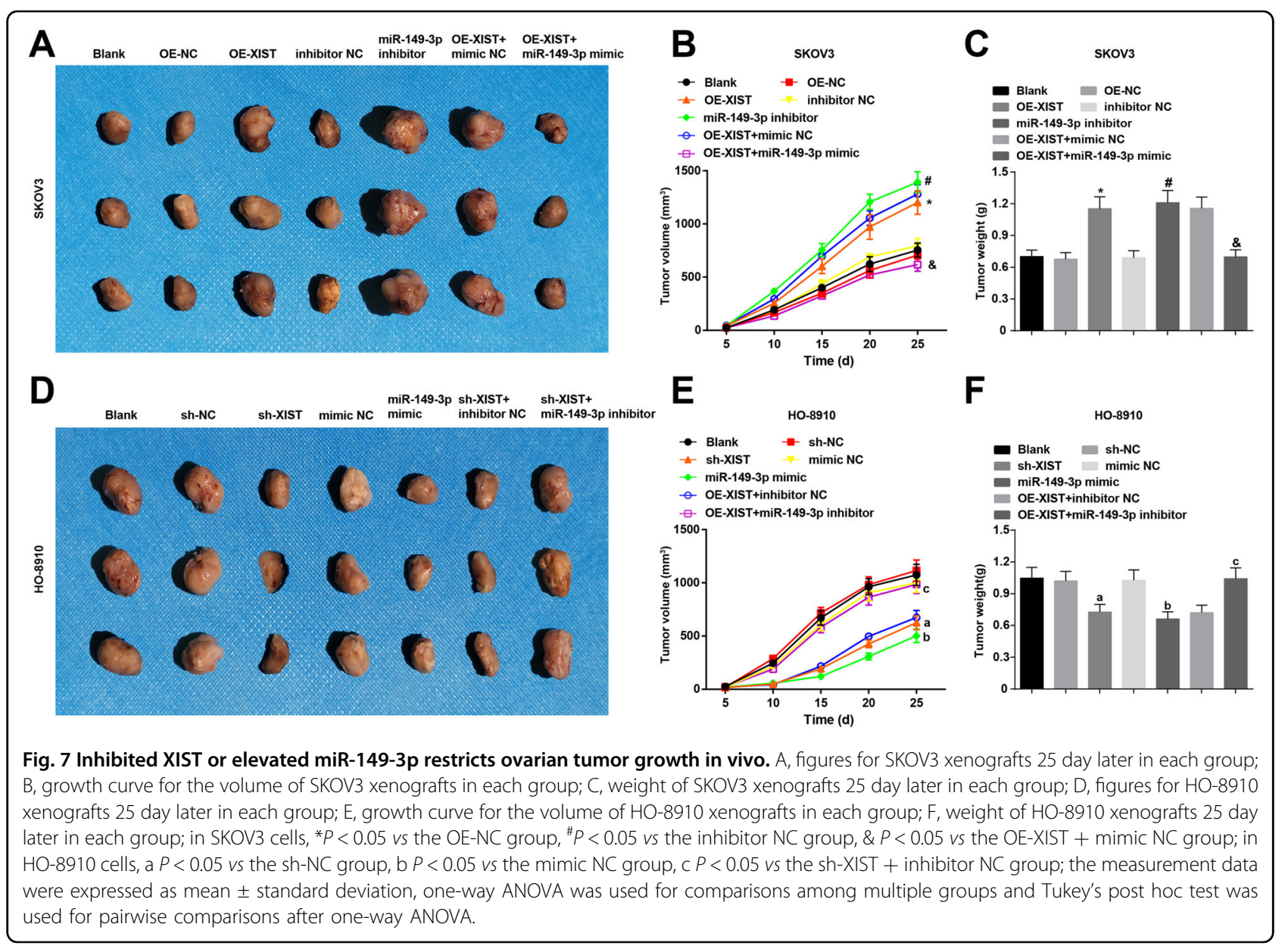


In addition, the $\mathrm{OC}$ cells were accordingly transfected with silenced/OE-XIST or miR-149-3p mimic/inhibitor, and the effects of altered XIST and miR-149-3p on OC cell phenotypes were observed. The outcomes reflected that reduction of XIST or elevation of miR-149-3p constrained proliferation and accelerated apoptosis of $\mathrm{OC}$ cells. In accordance with this finding, it has been recently reported that the knockdown of XIST decelerates proliferation and facilitates apoptosis of cervical cells (Siha and Hela cells ${ }^{24}$ and a publication has suggested that the restored miR-149 suppresses malignant behaviors of OC cells $^{14}$. We also found that the downregulated XIST elevated miR-149-3p to repress migration and invasion of OC cells. Similarly, XIST knockdown has been revealed to restrain migration and invasion of non-small cell lung cancer cells ${ }^{28}$, and the inhibitory role of elevated miR149-3p in migration and invasion of bladder cancer cells has also been verified ${ }^{29}$. Furthermore, the impacts of altered XIST and miR-149-3p on tumor growth in vivo was observed and we found that deleted XIST and promoted miR-149-3p restrained OC development in vivo. A similar finding by Liu et al. ${ }^{21}$ has implied that reduction of XIST restricts thyroid cancer cell proliferation in vivo and it has also been pointed out that miR-149 mimic inhibits tumor growth of $\mathrm{BC}$ xenografts ${ }^{30}$.

In conclusion, we have found that inhibition of IncRNA XIST and upregulation of miR-149-3p repressed the malignant behaviors of OC cells, which may contribute to exploration on OC therapeutic strategies. Great efforts remain to be done to further investigate the functional mechanisms of XIST on OC development.

\section{Acknowledgements}

We acknowledge the reviewers for their helpful comments on this paper.

\section{Funding}

This work was supported by National Natural Science Foundation of China (numbers 81672560, 81772773, and 81302275), Jiangsu Provincial Medical Youth Talent (numbers QNRC2016752 and QNRC2016753), and The Project of Jiangsu Provincial Maternal and Child Health Association (number FYX201709), and also by The Project of Suzhou Health and Family Planning Commission science project LCZX201705 and SYS2018030.

\footnotetext{
Author details

'Department of Obstetrics and Gynecology, The First Affiliated Hospital of Soochow University, Suzhou 215000 Jiangsu, China. ${ }^{2}$ Jiangsu Institute of Clinical Immunology, The First Affiliated Hospital of Soochow University, Suzhou 215000 Jiangsu, China. ${ }^{3}$ Jiangsu Key Laboratory of Clinical Immunology, Soochow University, Suzhou 215000 Jiangsu, China. ${ }^{4}$ Jiangsu Key Laboratory of Gastrointestinal Tumor Immunology, The First Affiliated Hospital of Soochow University, Suzhou 215000 Jiangsu, China. ${ }^{5}$ Department of Gynecology, The Second People's Hospital of Taizhou, Taizhou 225500 Jiangsu, China
}

\section{Author contributions}

Y.C. and F.S. finished study design. R.J., J.Z., J.W., Y.X., and He Zhang finished experimental studies. Hongyu Zhang, Y.G., F.F., Y.S., G.Z., L.F., and X.Z. finished data analysis. R.J. and Hongyu Zhang finished manuscript editing. All authors read and approved the final manuscript.

\section{Ethics statement}

This study was approved and supervised by the animal ethics committee of the First Affiliated Hospital of Soochow University. The treatment of animals in all experiments conforms to the ethical standards of experimental animals.

Conflict of interest

The authors declare that they have no conflict of interest.

\section{Publisher's note}

Springer Nature remains neutral with regard to jurisdictional claims in published maps and institutional affiliations.

Received: 28 May 2020 Revised: 30 November 2020 Accepted: 2 December 2020

Published online: 01 February 2021

\section{References}

1. Stewart, C., Ralyea, C. \& Lockwood, S. Ovarian cancer: an integrated review. Semin. Oncol. Nurs. 35, 151-156 (2019).

2. Muinao, T., Pal, M. \& Deka, H. P. Origins based clinical and molecular complexities of epithelial ovarian cancer. Int. J. Biol. Macromol. 118, 1326-1345 (2018).

3. Menon, U., Karpinskyj, C. \& Gentry-Maharaj, A. Ovarian cancer prevention and screening. Obstet. Gynecol. 131, 909-927 (2018).

4. Alharbi, M. et al. The potential role of miRNAs and exosomes in chemotherapy in ovarian cancer. Endocr. Relat. Cancer 25, R663-R685 (2018).

5. Jing, H., Qu, X., Liu, L. \& Xia, H. A novel long noncoding RNA (InCRNA), LL22NC03-N64E9.1, promotes the proliferation of lung cancer cells and is a potential prognostic molecular biomarker for lung cancer. Med Sci. Monit. 24, 4317-4323 (2018).

6. Zou, T., Wang, P. L., Gao, Y. \& Liang, W. T. Long noncoding RNA HOTTIP is a significant indicator of ovarian cancer prognosis and enhances cell proliferation and invasion. Cancer Biomark. 25, 133-139 (2019).

7. Zhao, X., Cheng, Z. \& Wang, J. Long noncoding RNA FEZF1-AS1 promotes proliferation and inhibits apoptosis in ovarian cancer by activation of JAKSTAT3 pathway. Med. Sci. Monit. 24, 8088-8095 (2018).

8. Chen, $Y$. et al. The long noncoding RNA XIST protects cardiomyocyte hypertrophy by targeting miR-330-3p. Biochem. Biophys. Res. Commun. 505, 807-815 (2018).

9. Ren, C. et al. Functions and mechanisms of long noncoding RNAs in ovarian cancer. Int. J. Gynecol. Cancer 25, 566-569 (2015).

10. Chen, X. et al. Up-regulated IncRNA XIST contributes to progression of cervical cancer via regulating miR-140-5p and ORC1. Cancer Cell Int. 19, 45 (2019).

11. Li, X. H., Ha, C. T. \& Xiao, M. MicroRNA-30 inhibits antiapoptotic factor Mcl-1 in mouse and human hematopoietic cells after radiation exposure. Apoptosis 21, 708-720 (2016).

12. Gong, M. et al. HDAC4 mutations cause diabetes and induce beta-cell FoxO1 nuclear exclusion. Mol. Genet. Genom. Med. 7, e602 (2019).

13. Liu, Y. et al. MicroRNA-214 suppresses ovarian cancer by targeting betacatenin. Cell Physiol. Biochem. 45, 1654-1662 (2018).

14. Sun, L., Zhai, R., Zhang, L. \& Zhao, S. MicroRNA-149 suppresses the proliferation and increases the sensitivity of ovarian cancer cells to cisplatin by targeting $X$ linked inhibitor of apoptosis. Oncol. Lett. 15, 7328-7334 (2018).

15. Li, Y. et al. Characterizing the landscape of peritoneal exosomal microRNAs in patients with ovarian cancer by high-throughput sequencing. Oncol. Lett. 17, 539-547 (2019).

16. Liu, Y. et al. Long non-coding RNA XIST contributes to osteoarthritis progression via miR-149-5p/DNMT3A axis. Biomed. Pharmacother. 128, 110349 (2020).

17. Zhang, H. Y. \& Sun, H. Up-regulation of Foxp3 inhibits cell proliferation, migration and invasion in epithelial ovarian cancer. Cancer Lett. 287, 91-97 (2010). 
18. Wolf, D. et al. The expression of the regulatory $T$ cell-specific forkhead box transcription factor FoxP3 is associated with poor prognosis in ovarian cancer Clin. Cancer Res. 11, 8326-8331 (2005).

19. Heintz, A. P. et al. Carcinoma of the fallopian tube. FIGO 26th Annual Report on the Results of Treatment in Gynecological Cancer. Int. J. Gynaecol. Obstet. 95, S145-S160 (2006).

20. Wang, J., Xu, W., He, Y., Xia, Q. \& Liu, S. LncRNA MEG3 impacts proliferation, invasion, and migration of ovarian cancer cells through regulating PTEN. Inflamm. Res. 67, 927-936 (2018).

21. Liu, H., Deng, H., Zhao, Y., Li, C. \& Liang, Y. LncRNA XIST/miR-34a axis modulates the cell proliferation and tumor growth of thyroid cancer through METPI3K-AKT signaling. J. Exp. Clin. Cancer Res. 37, 279 (2018).

22. Li, J. P. et al. miR-133a-3p/FOXP3 axis regulates cell proliferation and autophagy in gastric cancer. J. Cell Biochem. 121, 3392-3405 (2020).

23. Yang, M. et al. Long non-coding RNA FLJ33360 participates in ovarian cancer progression by sponging miR-30b-3p. Onco Targets Ther. 12, 4469-4480 (2019).

24. Zhu, H., Zheng, T., Yu, J., Zhou, L. \& Wang, L. LncRNA XIST accelerates cervical cancer progression via upregulating Fus through competitively binding with miR-200a. Biomed. Pharmacother. 105, 789-797 (2018).
25. Zhang, M. et al. miR-149-3p reverses CD8(+) T-cell exhaustion by reducing inhibitory receptors and promoting cytokine secretion in breast cancer cells. Open Biol. 9, 190061 (2019).

26. Yildirim, N. et al. Do tumor-infiltrating lymphocytes really indicate favorable prognosis in epithelial ovarian cancer? Eur. J. Obstet. Gynecol. Reprod. Biol. 215 55-61 (2017).

27. Schouten, P. C. et al. High XIST and low 53BP1 expression predict poor outcome after high-dose alkylating chemotherapy in patients with a BRCA1like breast cancer. Mol. Cancer Ther. 15, 190-198 (2016).

28. Qiu, H. B., Yang, K., Yu, H. Y. \& Liu, M. Downregulation of long non-coding RNA XIST inhibits cell proliferation, migration, invasion and EMT by regulating miR212-3p/CBLL1 axis in non-small cell lung cancer cells. Eur. Rev. Med. Pharm. Sci. 23, 8391-8402 (2019)

29. Yang, D., Du, G., Xu, A., Xi, X. \& Li, D. Expression of miR-149-3p inhibits proliferation, migration, and invasion of bladder cancer by targeting S100A4 Am. J. Cancer Res. 7, 2209-2219 (2017).

30. Dong, Y., Chang, C., Liu, J. \& Qiang, J. Targeting of GIT1 by miR-149* in breast cancer suppresses cell proliferation and metastasis in vitro and tumor growth in vivo. Onco Targets Ther. 10, 5873-5882 (2017) 